\title{
Ordered Water Layer on the Macroscopically Hydrophobic Fluorinated Polymer Surface and Its Ultrafast Vibrational Dynamics
}

Jiahui Zhang, Junjun Tan, Ruoqi Pei, Shuji Ye*, Yi Luo*

Hefei National Laboratory for Physical Sciences at the Microscale, Department of Chemical Physics, and Synergetic Innovation Center of Quantum Information \& Quantum Physics, University of Science and Technology of China, Hefei, 230026, China.

1. Film thickness and contact angle measurement.

2. Fitting of the SFG-VS signals.

3. Operations for IR pump- SFG probe experiments and data processing.

4. The SFG spectra of PTFE contacting with $\mathrm{D}_{2} \mathrm{O}$.

5. The SFG spectra of other polymer films exposed to air.

6. Table S2. The fitting parameters for the spectra in Figure 1.

7. The ppp SFG spectra of water at the PTFE/air surface in different humidity.

8. Orientational analysis of free $\mathrm{OH}$.

9. The fitting curve for the ppp spectrum in Figure 1a.

10. The reported frequency of the water clusters $(\mathrm{N}=2-6)$ in different matrix.

11. Four-level vibrational model used to extract the vibrational relaxation time of the ssp intensity decay of hydrogen-bonded $\mathrm{OH}$ groups.

12. The vibrational relaxation time of the ssp intensity decay with $\omega_{\text {pump }}=\omega_{\text {probe }}=\sim 3200$ $\mathbf{c m}^{-1}$ changes with humidity.

13. The SFG spectra at the PTFE/air surface in HOD atmosphere and the vibrational dynamics of HOD.

14. The relationship between the depth monitored by SFG and the vibrational relaxation time of the ssp intensity decay with $\omega_{\text {pump }}=\omega_{\text {probe }}=\sim 3200 \mathrm{~cm}^{-1}$.

15. The vibrational dynamics of dangling $\mathrm{OH}$ at the PTFE/air surface. 


\section{EXPERIMENTAL SECTION}

\section{Film thickness and contact angle measurement.}

The thickness of PTFE film was measured by USTC Center for Micro-and Nanoscale Research and Fabrication using SEMILAB GES-5E Spectroscopic Ellipsometry Analyzer, and the extracted data was analyzed by Spectroscopic Ellipsometry Analyzer (version 1.4.32). Contact angle measurement was carried out by a KSV CAM 200 contact angle goniometer at $24{ }^{\circ} \mathrm{C}$, and the volume of each $\mathrm{H}_{2} \mathrm{O}$ drop is controlled at $\sim 4 \mu \mathrm{L}$. The thickness of PTFE film is ca. $150 \mathrm{~nm}$, and shows hydrophobic character with $\mathrm{H}_{2} \mathrm{O}$ contact angle of $\sim 125^{\circ}$ (Table $\mathrm{S} 1$ ).

Table S1. Contact angle of water on PTFE film surface in different humidity

\begin{tabular}{ccccc}
\hline Relative humidity & $22.5 \%$ & $43.2 \%$ & $93.6 \%$ & air \\
\hline picture & 0 & $126^{\circ} \pm 2^{\circ}$ & $125^{\circ} \pm 1^{\circ}$ & $125^{\circ} \pm 1^{\circ}$ \\
contact angle & $126^{\circ} \pm 1^{\circ}$ & & & \\
\hline
\end{tabular}

\section{Fitting of the SFG-VS signals.}

The intensity of the SFG radiation is proportional to the square of the material's secondorder susceptibility $\left(\chi_{e f f}^{(2)}\right)$, the intensity of the input visible beam $\left(I_{1}\left(\omega_{v i s}\right)\right)$ and the intensity of the input IR beam $\left(I_{2}\left(\omega_{I R}\right)\right)$, as shown in Eq. $(\mathrm{S} 1),{ }^{1-5}$

$$
I\left(\omega_{S F G}\right) \propto\left|\chi_{e f f}^{(2)}\right|^{2} I_{1}\left(\omega_{V i s}\right) I_{2}\left(\omega_{I R}\right)
$$

where $\omega_{S F G}=\omega_{I R}+\omega_{v i s}$. For materials having inversion symmetry, the value of $\chi_{e f f}^{(2)}$ is zero. No SFG signal would be generated in the bulk, whereas signals appear at the surface and interface due to the breakage of inversion symmetry. $\chi_{e f}^{(2)} f$ is composed of two components: the effective surface nonlinear susceptibility $\left(\chi_{R}^{(2)}\right)$ and the non-resonant background $\left(\chi_{N R}^{(2)}\right)$. By properly tuning the frequency of the IR beam over the vibrational resonance of the surface/interface molecules, the value of $\chi_{R}^{(2)}$ can reach its maximum. $\chi_{e f f}^{(2)}$ is frequencydependent, as described in Eq. (S2),

$$
\chi_{e f f}^{(2)}(\omega)=\chi_{N R}^{(2)}+\sum_{v} \frac{A_{v}}{\omega-\omega_{v}+i \Gamma_{v}}
$$

where $A_{v}, \omega_{v}$, and $\Gamma_{v}$ refer to the strength, resonant frequency, and damping coefficient of the vibrational mode $(v)$, respectively. $A_{v}$ could be either positive or negative depending on the 
phase of the vibrational mode, and $A_{v}, \omega_{v}, \Gamma_{v}$ can be extracted by fitting the spectrum. We defined the effective peak strength $\left(\chi_{v}^{(2)}\right)$ as the fitting strength $A_{v}$ normalized by $\Gamma_{v}$

\section{Operations for IR pump- SFG probe experiments and data processing.}

The theoretical model and derived expressions for IR pump- SFG probe experimental analysis have been provided in detail in our previous articles. ${ }^{6-8}$ Followings are the experimental operations for time-resolved IR pump- SFG probe measurements.

The determination of time-zero. For the SFG probe, the time-zero was determined by monitoring the IR-visible sum-frequency signals from the $\mathrm{O}-\mathrm{H}$ vibration band $\mathrm{of}_{2} \mathrm{O}$ as a function of the delay between the IR probe and the visible pulses. For the IR pump- SFG probe, the time-zero and instrument response were determined by monitoring the third-order crosscorrelation of infrared-infrared visible sum-frequency signals from the $\mathrm{O}-\mathrm{H}$ vibration band of $\mathrm{H}_{2} \mathrm{O}$ as a function of the delay between the IR pump and IR probe pulses.

Spectra acquisition and data processing. A LABVIEW program was used to control the delay time between the IR pump and the IR probe, as well as to switch the pump IR on and off by controlling TOPAS shutter. For each delay time, a system consisted of chopper and galvanometric scanner was used to minimize the influence of laser fluctuations on the pumpon and pump-off spectra. Each spectrum has a collection time of $1.0 \mathrm{~s}$ (1000 laser shots) with 100 times accumulation. It takes $115.6 \mathrm{~s}$ (including the instrumental response time) to collect one spectrum. It takes $\sim 2.5 \mathrm{~h}$ to collect all of the transient spectra with the delay time ranging from -1 ps to 4 ps.

The obtained time-resolved IR pump- SFG probe spectra were measured as a function of the delay time $(\Delta \mathrm{t})$ between the IR pump and the SFG probe pair. The intensity decay of the vibrational modes presented in this study is given by the ratio between the integrated SFG intensities from $\omega_{v}-50 \mathrm{~cm}^{-1}$ to $\omega_{v}+50 \mathrm{~cm}^{-1}$ with and without the infrared pump. All the IR pumpSFG probe transients presented here were collected under pssp ( $p$-polarized infrared pump, spolarized SFG output, s-polarized visible input, p-polarized infrared probe) polarization combinations.

\section{SUPPLEMENTARY RESULTS AND DISCUSSIONS}

\section{The SFG spectra of PTFE contacting with $\mathrm{D}_{2} \mathrm{O}$.}


To find out the source of O-H signals at PTFE/air surface, we recorded the spectra when PTFE is in contact with bulk $\mathrm{D}_{2} \mathrm{O}$, as shown in Figure $\mathrm{S} 1$. No discernable O-H peaks can be found at PTFE/ $\mathrm{D}_{2} \mathrm{O}$ interface, meaning that the $\mathrm{O}-\mathrm{H}$ signals at PTFE/air surface all come from $\mathrm{H}_{2} \mathrm{O}$ molecules adsorbed at PTFE surface, rather than from $\mathrm{CaF}_{2} / \mathrm{PTFE}$ interface or PTFE matrix, or the impurity of the films. When PTFE surface is contacting with $\mathrm{D}_{2} \mathrm{O}$, surface $\mathrm{H}_{2} \mathrm{O}$ molecules would be replaced by O-D species and thus disappear in the spectrum.

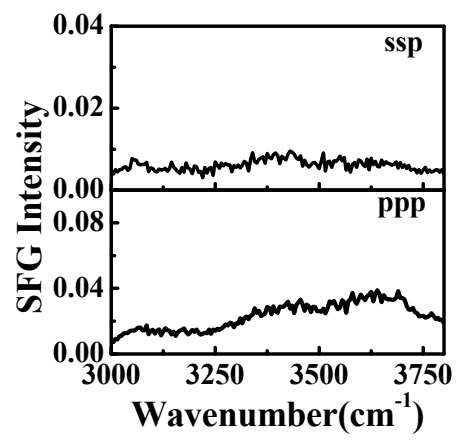

Figure S1. The SFG spectra of PTFE contacting with $\mathrm{D}_{2} \mathrm{O}$.

\section{The SFG spectra of other polymer films exposed to air.}

The SFG spectra of PS/air, PET/air and PVC/air were also examined, as shown in Figure $\mathrm{S} 2$. When these samples are exposed to air, no significant $\mathrm{O}-\mathrm{H}$ signals can be detected. That is to say, it is not a common phenomenon to observe ordered water molecules adsorbed on the surface of polymeric materials.

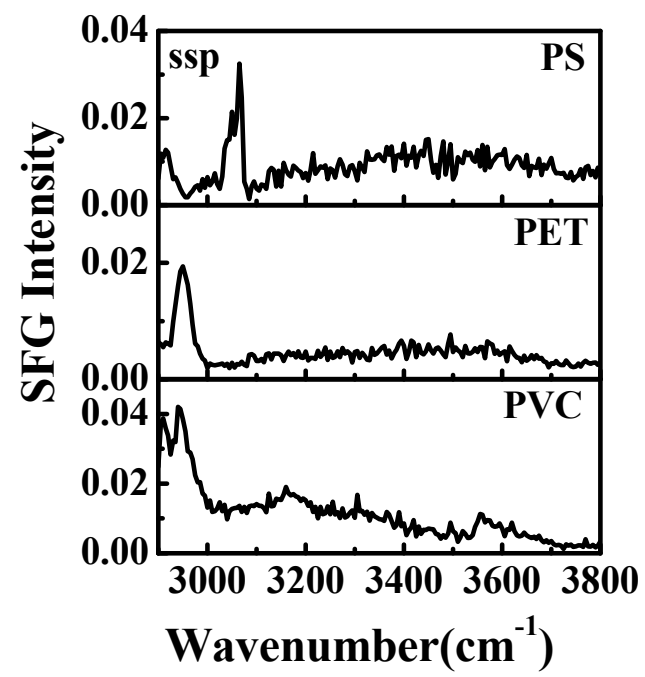


Figure S2. The ssp SFG spectra of other polymer films exposed to air.

6. Table S2. The fitting parameters for the spectra in Figure 1.

Table S2. The fitting parameters for the spectra in Figure 1

\begin{tabular}{|c|c|c|c|c|c|}
\hline \multirow{2}{*}{\multicolumn{2}{|c|}{ surface }} & PTFE/air, & PTFE/air, & $\mathrm{air} / \mathrm{H}_{2} \mathrm{O}$ & $\mathrm{PTFE} / \mathrm{H}_{2} \mathrm{O}$, \\
\hline & & ssp & ppp & ssp & $\operatorname{ssp}$ \\
\hline \multicolumn{2}{|c|}{ Non-resonance } & -0.0034 & -0.033 & 0.094 & -0.034 \\
\hline \multirow{3}{*}{ Peak1 } & $\mathrm{A}_{1}$ & 3.51 & 4.18 & -5.46 & 47.1 \\
\hline & $\omega_{1}\left(\mathrm{~cm}^{-1}\right)$ & 3280.8 & 3242.5 & 3239.4 & 3245.3 \\
\hline & $\Gamma_{1}\left(\mathrm{~cm}^{-1}\right)$ & 76.2 & 80.0 & 115.0 & 165.3 \\
\hline \multirow{3}{*}{ Peak2 } & $\mathrm{A}_{2}$ & 21.0 & 36.5 & -18.6 & 26.5 \\
\hline & $\omega_{2}\left(\mathrm{~cm}^{-1}\right)$ & 3432.5 & 3407.6 & 3454.0 & 3453.0 \\
\hline & $\Gamma_{2}\left(\mathrm{~cm}^{-1}\right)$ & 120.5 & 140.4 & 122.0 & 114.6 \\
\hline \multirow{3}{*}{ Peak3 } & $\mathrm{A}_{3}$ & 1.57 & 3.51 & 4.28 & 2.57 \\
\hline & $\omega_{3}\left(\mathrm{~cm}^{-1}\right)$ & 3681.3 & 3678.1 & 3693.1 & 3692.5 \\
\hline & $\Gamma_{3}\left(\mathrm{~cm}^{-1}\right)$ & 24.4 & 27.8 & 18.9 & 23.1 \\
\hline \multirow{3}{*}{ Peak4 } & $\mathrm{A}_{4}$ & - & - & 7.97 & - \\
\hline & $\omega_{4}\left(\mathrm{~cm}^{-1}\right)$ & - & - & 3129.7 & - \\
\hline & $\Gamma_{4}\left(\mathrm{~cm}^{-1}\right)$ & - & - & 100.0 & - \\
\hline
\end{tabular}

7. The ppp SFG spectra of water at the PTFE/air surface in different humidity. 


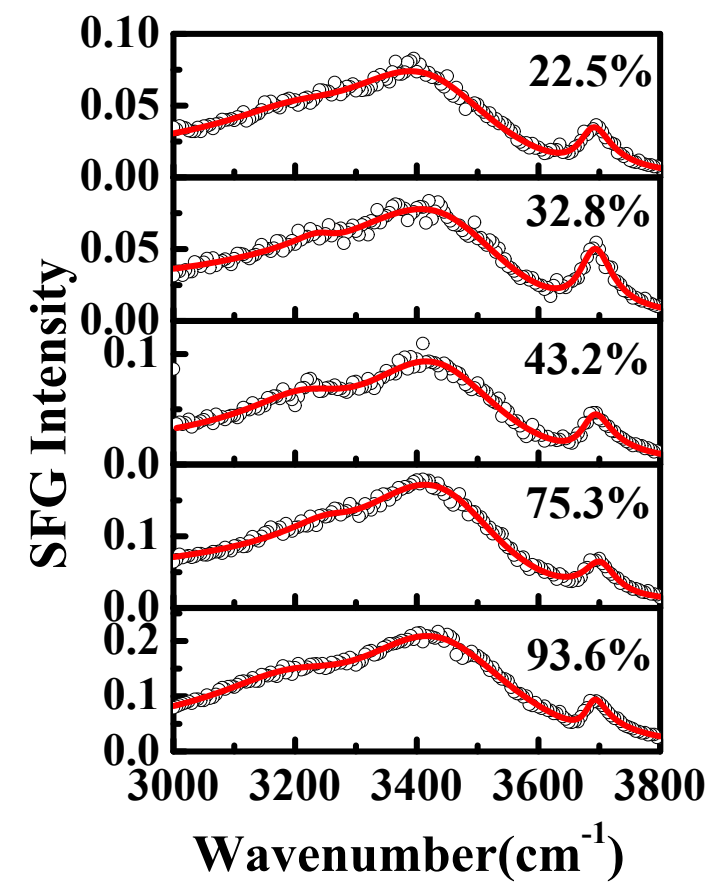

Figure S3. The ppp SFG spectra of water at the PTFE/air surface in different humidity.

Figure S3 shows the ppp spectra of PTFE/air surface in different humidity. Similar to the spectrum at PTFE/ $\mathrm{H}_{2} \mathrm{O}$ interface, the ppp spectra at PTFE/air surface in different humidity are mainly composed of three resonant peaks. Besides, the fitting strength of $\sim 3440 \mathrm{~cm}^{-1}$ peak builds up as the environmental R.H. increases (shown in Figure 2b), and the humidity-

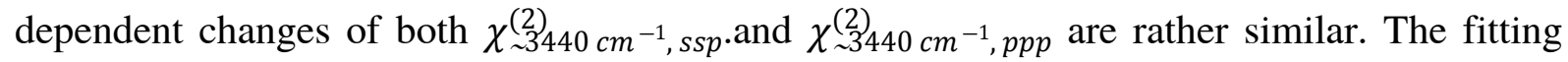
parameters for ssp and ppp spectra of PTFE/air surface in different humidity are given in Table S3 and S4, respectively.

Table S3. The fitting parameters for the spectra in Figure 2a

\begin{tabular}{|c|c|c|c|c|c|c|}
\hline \multicolumn{2}{|c|}{ humidity } & $22.5 \%$ & $32.8 \%$ & $43.2 \%$ & $75.3 \%$ & $93.6 \%$ \\
\hline \multicolumn{2}{|c|}{ Non-resonance } & -0.018 & -0.020 & -0.017 & -0.049 & -0.056 \\
\hline \multirow{2}{*}{ Peak1 } & $\mathrm{A}_{1}$ & 3.99 & 3.53 & 4.13 & 4.51 & 5.54 \\
\cline { 2 - 7 } & $\omega_{1}\left(\mathrm{~cm}^{-1}\right)$ & 3282.7 & 3276.7 & 3275.8 & 3273.1 & 3261.4 \\
\hline
\end{tabular}




\begin{tabular}{|c|c|c|c|c|c|c|}
\hline & $\Gamma_{1}\left(\mathrm{~cm}^{-1}\right)$ & 80.0 & 80.0 & 80.0 & 80.0 & 80.0 \\
\hline \multirow{3}{*}{ Peak2 } & $\mathrm{A}_{2}$ & 23.5 & 25.5 & 26.5 & 36.9 & 45.6 \\
\cline { 2 - 7 } & $\omega_{2}\left(\mathrm{~cm}^{-1}\right)$ & 3438.3 & 3449.5 & 3440.8 & 3451.8 & 3456.8 \\
\cline { 2 - 7 } & $\Gamma_{2}\left(\mathrm{~cm}^{-1}\right)$ & 122.2 & 115.6 & 116.5 & 122.7 & 136.9 \\
\hline \multirow{3}{*}{ Peak3 } & $\mathrm{A}_{3}$ & 1.51 & 2.84 & 2.43 & 2.30 & 1.87 \\
\cline { 2 - 7 } & $\omega_{3}\left(\mathrm{~cm}^{-1}\right)$ & 3681.4 & 3680.9 & 3683.1 & 3688.4 & 3690.5 \\
\cline { 2 - 7 } & $\Gamma_{3}\left(\mathrm{~cm}^{-1}\right)$ & 27.8 & 33.2 & 34.6 & 28.1 & 20.9 \\
\hline
\end{tabular}

Table S4. The fitting parameters for the spectra in Figure S3

\begin{tabular}{|c|c|c|c|c|c|c|}
\hline \multicolumn{2}{|c|}{ humidity } & $22.5 \%$ & $32.8 \%$ & $43.2 \%$ & $75.3 \%$ & $93.6 \%$ \\
\hline \multicolumn{2}{|c|}{ offset } & 0.0036 & 0.0047 & 0.0060 & 0.010 & 0.0061 \\
\hline \multicolumn{2}{|c|}{ Non-resonance } & -0.075 & -0.077 & -0.064 & -0.105 & -0.103 \\
\hline \multirow{3}{*}{ Peak1 } & $\mathrm{A}_{1}$ & 2.06 & 2.25 & 4.76 & 3.35 & 5.33 \\
\hline & $\omega_{1}\left(\mathrm{~cm}^{-1}\right)$ & 3232.8 & 3238.1 & 3241.9 & 3250.1 & 3217.5 \\
\hline & $\Gamma_{1}\left(\mathrm{~cm}^{-1}\right)$ & 80.0 & 80.0 & 80.0 & 80.0 & 80.0 \\
\hline \multirow{3}{*}{ Peak2 } & $\mathrm{A}_{2}$ & 39.0 & 40.2 & 39.6 & 58.1 & 76.4 \\
\hline & $\omega_{2}\left(\mathrm{~cm}^{-1}\right)$ & 3435.5 & 3452.0 & 3442.9 & 3449.9 & 3451.8 \\
\hline & $\Gamma_{2}\left(\mathrm{~cm}^{-1}\right)$ & 162.8 & 166.1 & 143.2 & 157.4 & 180.8 \\
\hline \multirow{3}{*}{ Peak3 } & $\mathrm{A}_{3}$ & 2.97 & 3.76 & 2.94 & 2.84 & 2.79 \\
\hline & $\omega_{3}\left(\mathrm{~cm}^{-1}\right)$ & 3690.6 & 3691.6 & 3689.1 & 3692.9 & 3692.1 \\
\hline & $\Gamma_{3}\left(\mathrm{~cm}^{-1}\right)$ & 28.5 & 28.2 & 25.2 & 23.4 & 23.8 \\
\hline
\end{tabular}

\section{Orientational analysis of free $\mathrm{OH}$.}


The detailed method to determine the orientation of interfacial free $\mathrm{OH}$ at $\sim 3700 \mathrm{~cm}^{-1}$ has already been given in the references. ${ }^{9-11}$ The average tilt angle of a functional group can be determined by relating the SFG non-zero susceptibility tensor element $\chi_{i j k}(i, j, k=x, y, z)$ and molecular hyperpolarizability $\beta_{l m n}(l, m, n=a, b, c)$.

The components of $\chi_{e f f, s s p}^{(2)}$ and $\chi_{e f f, p p p}^{(2)}$ can be expressed as Eqs.(S3)-(S4) in the lab coordinate system in which the $\mathrm{z}$-axis is defined along the surface normal and the $\mathrm{x}$-axis in the incident plane.

$$
\begin{aligned}
\chi_{e f f, s s p}^{(2)}= & L_{y y}\left(\omega_{S F G}\right) L_{y y}\left(\omega_{v i s}\right) L_{z z}\left(\omega_{I R}\right) \sin \beta_{I R} \chi_{y y z}^{(2)} \\
\chi_{e f f, p p p}^{(2)}= & -L_{x x}\left(\omega_{S F G}\right) L_{x x}\left(\omega_{v i s}\right) L_{z z}\left(\omega_{I R}\right) \cos \beta_{S F G} \cos \beta_{v i s} \sin \beta_{I R} \chi_{x x z}^{(2)} \\
& -L_{x x}\left(\omega_{S F G}\right) L_{z z}\left(\omega_{v i s}\right) L_{x x}\left(\omega_{I R}\right) \cos \beta_{S F G} \sin \beta_{v i s} \cos \beta_{I R} \chi_{x z x}^{(2)} \\
& +L_{z z}\left(\omega_{S F G}\right) L_{x x}\left(\omega_{v i s}\right) L_{x x}\left(\omega_{I R}\right) \sin \beta_{S F G} \cos \beta_{v i s} \cos \beta_{I R} \chi_{z x x}^{(2)} \\
& +L_{z z}\left(\omega_{S F G}\right) L_{z z}\left(\omega_{v i s}\right) L_{z z}\left(\omega_{I R}\right) \sin \beta_{S F G} \sin \beta_{v i s} \sin \beta_{I R} \chi_{z z Z}^{(2)}
\end{aligned}
$$

where $L_{i i}$ is the diagonal element of the Fresnel factor, $\omega_{S F G}\left(\beta_{S F G}\right), \omega_{v i s}\left(\beta_{v i s}\right)$, and $\omega_{I R}\left(\beta_{I R}\right)$ refer to the frequencies (incident angle) of SFG, visible light and IR light, respectively. ${ }^{1-5}$

The Fresnel factors can be obtained by the following expressions,

$$
\begin{aligned}
& L_{x x}\left(\omega_{i}\right)=\frac{2 n_{1}\left(\omega_{i}\right) \cos \gamma_{i}}{n_{1}\left(\omega_{i}\right) \cos \gamma_{i}+n_{2}\left(\omega_{i}\right) \cos \beta_{i}} \\
& L_{y y}\left(\omega_{i}\right)=\frac{2 n_{1}\left(\omega_{i}\right) \cos \beta_{i}}{n_{1}\left(\omega_{i}\right) \cos \beta_{i}+n_{2}\left(\omega_{i}\right) \cos \gamma_{i}}
\end{aligned}
$$

$L_{z Z}\left(\omega_{i}\right)=\frac{2 n_{2}\left(\omega_{i}\right) \cos \beta_{i}}{n_{1}\left(\omega_{i}\right) \cos \gamma_{i}+n_{2}\left(\omega_{i}\right) \cos \beta_{i}} *\left(\frac{n_{1}\left(\omega_{i}\right)}{n^{\prime}\left(\omega_{i}\right)}\right)^{2}, \quad n^{\prime}\left(\omega_{i}\right)=\frac{n_{1}\left(\omega_{i}\right)+n_{2}\left(\omega_{i}\right)}{2}$

where $n_{i}$ is the reflective index of the bulk medium, $n^{\prime}$ is the effective reflective index of the interface, $\beta_{i}$ is the incident angle, and $\gamma_{i}$ is the reflective angle when the light comes into medium 2.12 Here, mediums 1 and 2 refer to the PTFE and gas-phase, respectively. The refractive index of PTFE was set to $1.29\left(n_{1}(S F G)=n_{1}(v i s)=n_{1}(I R)=1.29\right),{ }^{13}$ and the gasphase refractive index was set to $1\left(n_{2}(S F G)=n_{2}(\right.$ vis $\left.)=n_{2}(I R)=1\right)$.

After considering the Fresnel coefficient constants under the experimental geometry in our lab, Eqs. (S3)-(S4) can be simplified into Eqs. (S8)-(S9),

$\chi_{e f f, s s p}^{(2)}=1.2745 \chi_{y y z}^{(2)}$

$\chi_{e f f, p p p}^{(2)}=-0.1357 \chi_{x x z}^{(2)}+1.1022 \chi_{z Z z}^{(2)}$

For $\mathrm{C}_{\infty \mathrm{V}}$ symmetry of free $\mathrm{OH}, \chi_{x x z}^{(2)}=\chi_{y y z}^{(2)}$. The $\chi_{y y z}^{(2)}$ and $\chi_{z z z}^{(2)}$ susceptibility components are the main contributors to the ssp and ppp signals, respectively. The dependence of $\chi_{y y z}^{(2)}$ and 
$\chi_{Z Z Z}^{(2)}$ susceptibility components on the molecular hyperpolarizability averaged over the azimuthal angles can be described by Eqs. (S10)-(S11).

$\chi_{x x z}^{(2), s s}=\chi_{y y z}^{(2), s s}=\frac{1}{2} N_{s} \beta_{c c c}\left[(1+R)<\cos \theta>-(1-R)<\cos ^{3} \theta>\right]$

$\chi_{z Z Z}^{(2), s s}=N_{s} \beta_{c c c}\left[R<\cos \theta>+(1-R)<\cos ^{3} \theta>\right]$

Combing Eqs. (S8)-(S11), we have the relationship at PTFE/air surface,

$\frac{\chi_{e f f, p p p}^{(2)}}{\chi_{e f f, s s p}^{(2)}}=-0.1065+1.7296 * \frac{R<\cos \theta>+(1-R)<\cos ^{3} \theta>}{(1+R)<\cos \theta>-(1-R)<\cos ^{3} \theta>}$

In terms of the references, ${ }^{10,14} \mathrm{R}={ }^{\beta_{a a c}} / \beta_{c c c}=\beta_{b b c} / \beta_{c c c}=0.32$.

In theory, the value of the averaged tilt angle of the dangling O-H group depends on the assumption of a functional form. Currently, functional forms of a narrow Gaussian- ${ }^{11}$ stepwiseshaped, ${ }^{9}$ or exponentially shaped distribution ${ }^{15}$ have been adopted. Assuming that the orientational distribution is a $\delta$-function, we can get the relationship between $\frac{\chi_{\text {eff,ppp }}^{(2)}}{\chi_{e f f, s p p}^{(2)}}$ and $\theta_{\text {free } \mathrm{OH}}$, as presented in Figure S4. It needs to mention that although different distribution could give different tilt angle, the tilt angle keeps almost constant for a certain distribution at R.H. $>30 \%$. Here we adopt a $\delta$-distribution, the tilt angle of dangling $\mathrm{OH}$ bond is $36^{\circ}\left( \pm 4^{\circ}\right)$ at R.H. $>30 \%$ (Figure 2c).

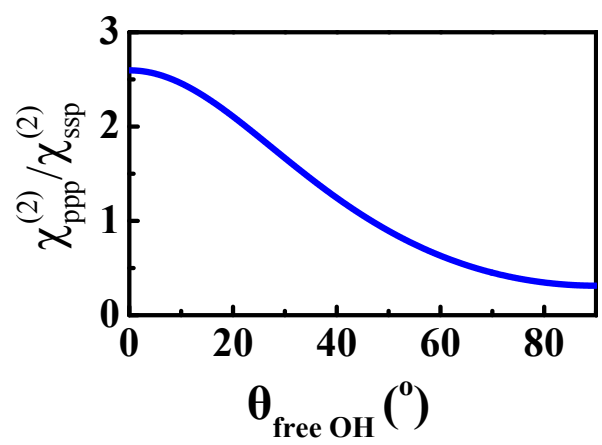

Figure S4. Relationship between $\chi_{p p p}^{(2)}($ free $O H) / \chi_{s S p}^{(2)}($ free $O H)$ and $\theta_{\text {free } O H}$ for the PTFE/air surface.

\section{The fitting curve for the ppp spectrum in Figure 1a.}




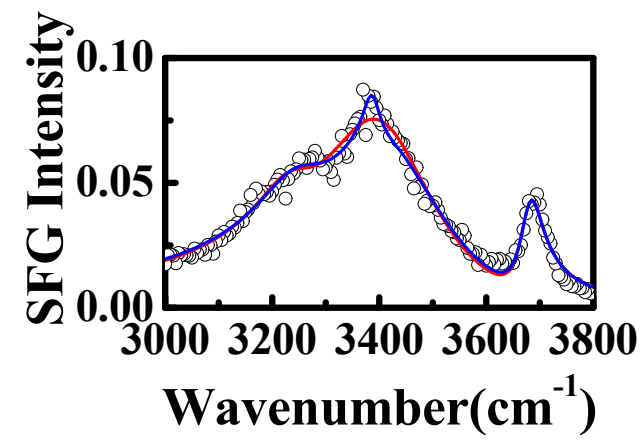

Figure S5. The fitting curve for the ppp spectrum in Figure 1a. Red curve is fitted using three peaks at $3243,3408,3678 \mathrm{~cm}^{-1}$. Blue curve is fitted using four peaks at $3243,3390\left(\Gamma=20 \mathrm{~cm}^{-}\right.$ $\left.{ }^{1}\right), 3408,3678 \mathrm{~cm}^{-1}$.

\section{The reported frequency of the water clusters $(\mathrm{N}=2-6)$ in different matrix.}

Table S5 and S6 present the frequency of the water clusters $(\mathrm{N}=2-6)$ given by previous experimental and theoretical studies. The frequency of tetramer structure in different environments all falls in the range of $3360-3440 \mathrm{~cm}^{-1}$, while there are several-ten wavenumber differences between water tetramer and other water clusters. Therefore, we tentatively assign the peaks observed at $\sim 3397 \mathrm{~cm}^{-1}$ in the ssp spectrum and at $3381 \mathrm{~cm}^{-1}$ in the ppp spectrum in Figure $3 \mathrm{a}$ to the tetramer water structure.

Table S5. The experimental frequency of the water clusters $(\mathrm{N}=2-6)$ in different matrix

\begin{tabular}{|c|c|c|c|c|c|c|}
\hline \multirow{2}{*}{ Matrix } & \multicolumn{5}{|c|}{ Frequency $\left(\mathrm{cm}^{-1}\right)$ of size N } & \multirow{2}{*}{ Ref. } \\
\cline { 2 - 6 } & 2 & 3 & 4 & 5 & 6 & \\
\hline $288 \mathrm{~K}$ bath 4 atm He & 3600 & 3545 & & & & 16 \\
\hline$\left[\mathrm{Co}(1,10 \text {-phenanthroline })_{2}\left(\mathrm{NO}_{3}\right)\right]$, room T & & & 3367 & & & 17 \\
\hline Cyano-bridged trimetallic complex T=22 ${ }^{\circ} \mathrm{C}$ & & & & & 3300 & 18 \\
\hline Gas phase in He at 3.5 bar & 3601 & 3533 & 3416 & 3360 & 3220 & 19,20 \\
\hline In a pulsed supersonic expansion & 3600 & 3532 & 3416 & 3360 & 3220 & 20,21 \\
\hline In helium droplets & & & 3395 & & & 22 \\
\hline In Ar matrix & & & 3373 & 3330 & 3211 & 23,24 \\
\hline In hybrid metal-organic host & & & 3388 & & & 25 \\
\hline In Liquid He & 3597 & 3529 & 3394 & 3353 & 3335 & 26 \\
\hline In Para-H & & 3544 & & & 3229 & \\
\hline
\end{tabular}




\begin{tabular}{|c|c|c|c|c|c|c|}
\hline & & 3518 & & & & \\
\hline In CCl $_{4}(\mathrm{~T}=296 \mathrm{~K})$ & 3620 & & & & & 28 \\
\hline In tetramethylammonium $\left(\mathrm{TMA}^{+}\right)$ & 3549 & & & & & \\
\hline In phenyltrimethylammonium $\left(\mathrm{PTMA}^{+}\right)$ & & 3488 & 3438 & & 3228 & 29 \\
\hline In $\mathrm{CCl}_{4}$ & $3615-$ & $3535-$ & $3395-$ & & $3221-$ & 30 \\
\hline In nano-structured CaHAs at $298 \mathrm{~K}$ & 3616 & 3587 & 3433 & & 3254 & \\
\hline In $\mathrm{Ne}$ & 3591 & & 3439 & & & 30 \\
\hline In $\mathrm{Ar}$ & & & 3383 & 3345 & 3224 & \\
\hline In Para- $\mathrm{H}_{2}$ & & & 3372 & 3330 & 3211 & 24 \\
\hline
\end{tabular}

Table S6. The calculated frequency of the water clusters $(\mathrm{N}=2-6)$ in different matrix

\begin{tabular}{|c|c|c|c|c|c|c|}
\hline \multirow{2}{*}{ Calculation method } & \multicolumn{5}{|c|}{ Frequency $\left(\mathrm{cm}^{-1}\right)$ of size N } & \multirow{2}{*}{ Ref. } \\
\cline { 2 - 7 } & 2 & 3 & 4 & 5 & 6 & \\
\hline MP2 (fc)/aug-cc-pVDZ & 3704 & 3573 & 3391 & 3354 & 3344 & 26 \\
\hline CCSD(T)/aug-cc-pVDZ & 3593 & 3529 & 3389 & 3350 & 3335 & 22 \\
\hline MP2 (full)6-311+G(d,2p) & 3585 & $\begin{array}{c}3524.5 \\
3529.8\end{array}$ & 3372.5 & 3329.0 & 3311.1 & 31 \\
\hline B3LYP/6-311++G** & & 3559 & 3374 & 3328 & 3317 & 33 \\
\hline B3LYP-D3/6-311++G** & & 3556.1 & 3372.73 & & & 34 \\
\hline
\end{tabular}

\section{Four-level vibrational model used to extract the vibrational relaxation time of the ssp intensity decay of hydrogen-bonded $\mathrm{OH}$ groups.}

The ultrafast vibrational dynamics of bulk and interfacial water are usually described by a well-established four-level vibrational model. ${ }^{35-38}$ As shown in Figure S6, upon the IR pump, $\mathrm{O}-\mathrm{H}$ stretch is excited from ground state $(v=0)$ to its first vibrational state $(v=1)$. This excitation leads to an apparent bleach of the signal. Afterwards, the energy of the O-H stretch mode at excited state transfers to an intermediate state $\left(v=v^{*}\right)$ with a relaxation time of $T_{1}$ attributed to the vibrational lifetime. The intermediate state is a mixed state involving the 
overtone of bending or the hydrogen bonding. ${ }^{35,39,40}$ Then, the energy of the O-H stretch mode at intermediate state further transfers to a hot ground state $(v=v * *)$ with a relaxation time of $\tau_{\text {eq }}$ attributed to thermalization process.

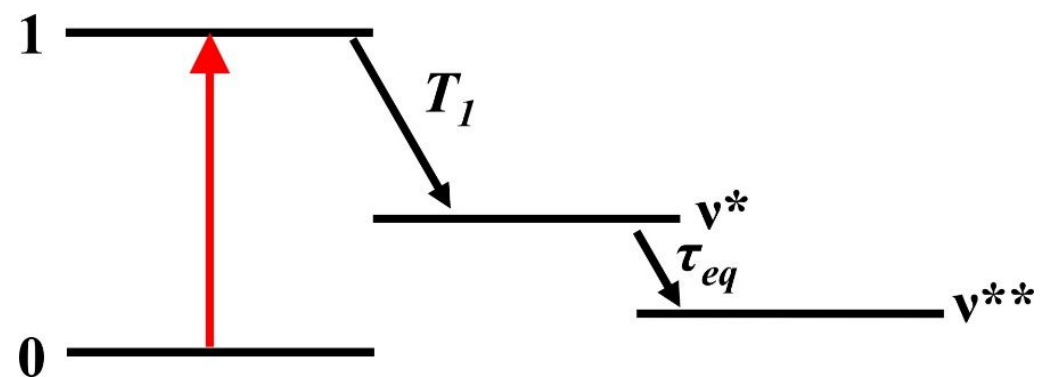

Figure S6. The four-level vibrational model used for analyzing the vibrational dynamics of water.

Below are the four coupled differential equations used to describe the transfer of population in each state:

$\frac{d N_{0}(t)}{d t}=-\sigma I_{\text {pump }}(t)\left[N_{0}(t)-N_{1}(t)\right]$

$\frac{d N_{1}(t)}{d t}=\sigma I_{\text {pump }}(t)\left[N_{0}(t)-N_{1}(t)\right]-\frac{N_{1}(t)}{T_{1}}$

$\frac{d N_{v^{*}}(t)}{d t}=\frac{N_{1}(t)}{T_{1}}-\frac{N_{v^{*}}(t)}{\tau_{e q}}$

$\frac{d N_{v^{* *}}(t)}{d t}=\frac{N_{v^{*}}(t)}{\tau_{e q}}$

where $N_{0}, N_{1}, N_{v^{*}}$ and $N_{v^{* *}}$ are the populations in the $v=0,1, v^{*}, v^{* *}$ states, respectively. $\sigma$ is the infrared absorption cross section of the vibrational ground state $(v=0)$, and $I_{\text {pump }}(t)$ is the Gaussian temporal intensity profile of the pump pulse.

To model our experimental data, Eqs. (S13)-(S16) are substituted into Eq. (S17). It is noted that, due to the large vibrational anharmonicity of water, the frequency of the O-H stretch mode at excited state is shifted out of our experimental window. So there is no signal contribution from the excited state $(v=1)$. Also with the assumption that $v^{*}$ state does not contribute to the response, we get Eq. (S17):

$\frac{I_{\text {pump on }}}{I_{\text {pump off }}}=\operatorname{IRF}(\mathrm{t}) *\left[\frac{N_{0}(t)-N_{1}(t)}{N_{0}}+\frac{\beta_{v^{* *} N_{v^{* *}}(t)}}{\beta_{0} N_{0}}\right]^{2}$

where $\beta_{0}$ and $\beta_{v^{* *}}$ are the molecular hyperpolarizabilities of $\mathrm{OH}$ oscillators in the vibrational ground state $(v=0)$ and the "hot" ground state $\left(v=v^{* *}\right), \operatorname{IRF}(\mathrm{t})$ refers to the Gaussian profile of instrument response function.

Based on proper approximation and boundary conditions, Eq. (S17) can be written into: 


$$
\begin{aligned}
\frac{I_{\text {pump on }}}{I_{\text {pump off }}=\operatorname{IRF}(\mathrm{t})} *\left\{1-2\left(1-\sqrt{S_{0}}\right) e^{-\frac{t-t_{0}}{T_{1}}}+2(\sqrt{1+\Delta S}-1)\left(1-e^{-\frac{t-t_{0}}{\tau_{e q}}}\right)\right. \\
+\left(1-\sqrt{S_{0}}\right)^{2} e^{-\frac{2\left(t-t_{0}\right)}{T_{1}}}-2\left(1-\sqrt{S_{0}}\right)(\sqrt{1+\Delta S}-1) e^{-\frac{t-t_{0}}{T_{1}}}\left(1-e^{-\frac{t-t_{0}}{\tau_{e q}}}\right) \\
\left.+(\sqrt{1+\Delta S}-1)^{2}\left(1-e^{-\frac{t-t_{0}}{\tau_{e q}}}\right)^{2}\right\}
\end{aligned}
$$

where $t_{0}$ is the moment when the SFG response reaches its maximum bleach, $S_{0}$ is the SFG response at $t=t_{0}, \Delta S$ is signal offset at long delay times. According to previous studies on IR pump- SFG probe measurements for $\mathrm{H}_{2} \mathrm{O}$, the value of $\tau_{e q}$ is $700 \mathrm{fs} .{ }^{35,36}$

As can be seen in Figure 4a, the value of $\Delta S$ is small, so Eq. (S18) can be further simplified as:

$$
\begin{gathered}
\frac{I_{\text {pump on }}}{I_{\text {pump off }}}=\operatorname{IRF}(\mathrm{t}) *\left\{1-2\left(1-\sqrt{S_{0}}\right) e^{-\frac{t-t_{0}}{T_{1}}}+2(\sqrt{1+\Delta S}-1)\left(1-e^{-\frac{t-t_{0}}{\tau_{e q}}}\right)+\left(1-\sqrt{S_{0}}\right.\right. \\
)^{2} e^{-\frac{2\left(t-t_{0}\right)}{T_{1}}}
\end{gathered}
$$

12. The vibrational relaxation time of the ssp intensity decay with $\omega_{\text {pump }}=\omega_{\text {probe }}=\sim 3200$ $\mathrm{cm}^{-1}$ changes with humidity.
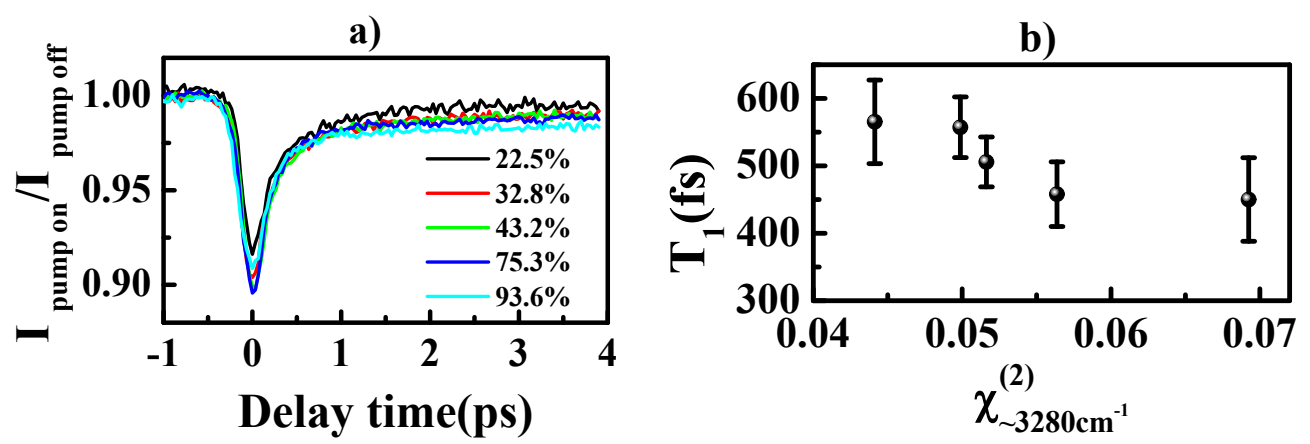

Figure S7. a) The ssp intensity decay of the strongly H-bonded band at the PTFE/air interface with $\omega_{\text {pump }}=\omega_{\text {probe }}=\sim 3200 \mathrm{~cm}^{-1}$ at different relative humidity. b) The relaxation time $\left(T_{1}\right)$ changes with the fitting amplitude of the $\sim 3280 \mathrm{~cm}^{-1}$ peak in the ssp spectra $\left(\tau_{\text {eq }}=700 \mathrm{fs}\right)$.

\section{The SFG spectra at the PTFE/air surface in HOD atmosphere and the vibrational dynamics of HOD.}

The SFG spectra in Figure S8a were measured when the PTFE surface is exposing to the HOD atmosphere, which was prepared by the mixture of $\mathrm{H}_{2} \mathrm{O} / \mathrm{D}_{2} \mathrm{O}$ with a volume ratio of 1:4. Figure S8b is the ssp intensity decay of the strongly H-bonded band at the PTFE/air interface with $\omega_{\text {pump }}=\omega_{\text {probe }}=\sim 3200 \mathrm{~cm}^{-1} . T_{1}$ is deduced to be $550 \pm 72 \mathrm{fs}\left(\tau_{\text {eq }}=700 \mathrm{fs}\right)$ for the PTFE/air surface in HOD atmosphere. This lifetime is close to the result when the PTFE surface is 
exposing to the $\mathrm{H}_{2} \mathrm{O}$ atmosphere at R.H. $\leq 35 \%$ (Figure 4c). But it is a little larger than the value (450 $\pm 70 \mathrm{fs}$ ) when the PTFE surface is exposing to the $\mathrm{H}_{2} \mathrm{O}$ atmosphere (Figure S8b, bottom panel), in which $\mathrm{H}_{2} \mathrm{O} / \mathrm{D}_{2} \mathrm{O}$ mixture(volume ratio of 1:4) was replaced by the pure $\mathrm{H}_{2} \mathrm{O}$, indicating the vibrational coupling at R.H. $\leq 35 \%$ is not very strong.
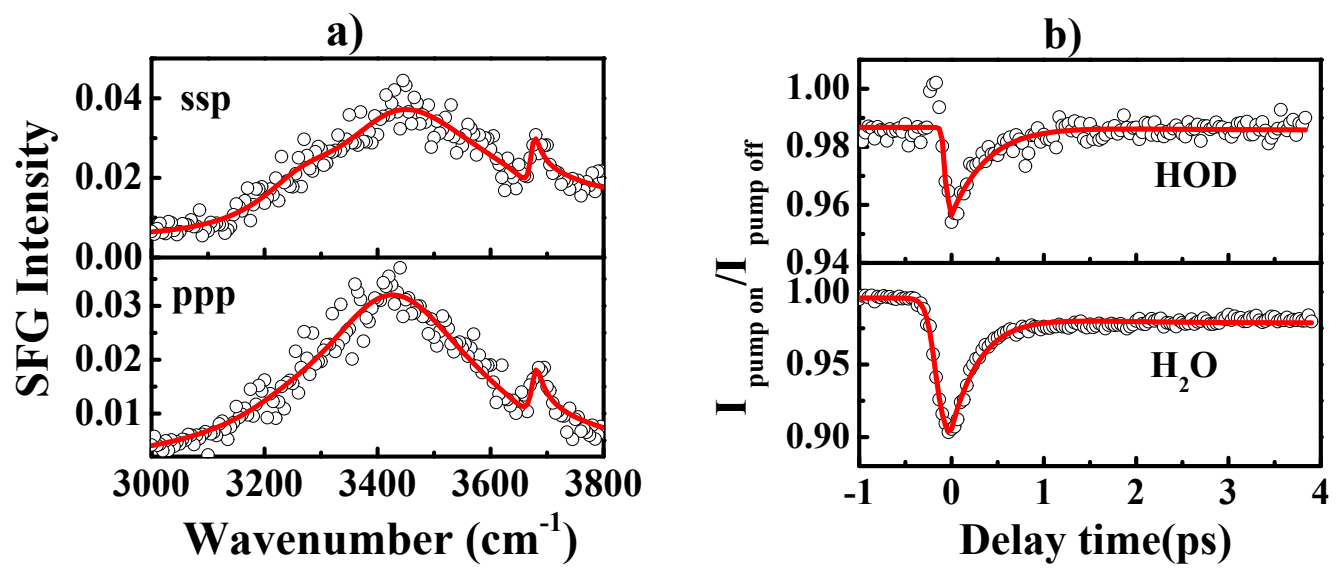

Figure S8. a) The ssp and ppp SFG spectra in the OH stretching region at the PTFE/air surface in HOD atmosphere. b) The ssp intensity decay of the strongly H-bonded band at the PTFE/air interface with $\omega_{\text {pump }}=\omega_{\text {probe }}=\sim 3200 \mathrm{~cm}^{-1}$, top panel: in the atmosphere of $\mathrm{H}_{2} \mathrm{O} / \mathrm{D}_{2} \mathrm{O}$ mixture atmosphere; bottom panel: atmosphere of the $\mathrm{H}_{2} \mathrm{O} / \mathrm{D}_{2} \mathrm{O}$ mixture(volume ratio of 1:4) was replaced by the pure $\mathrm{H}_{2} \mathrm{O}$.

\section{The relationship between the depth monitored by SFG and the vibrational relaxation} time of the ssp intensity decay with $\omega_{\text {pump }}=\omega_{\text {probe }}=\sim 3200 \mathrm{~cm}^{-1}$.

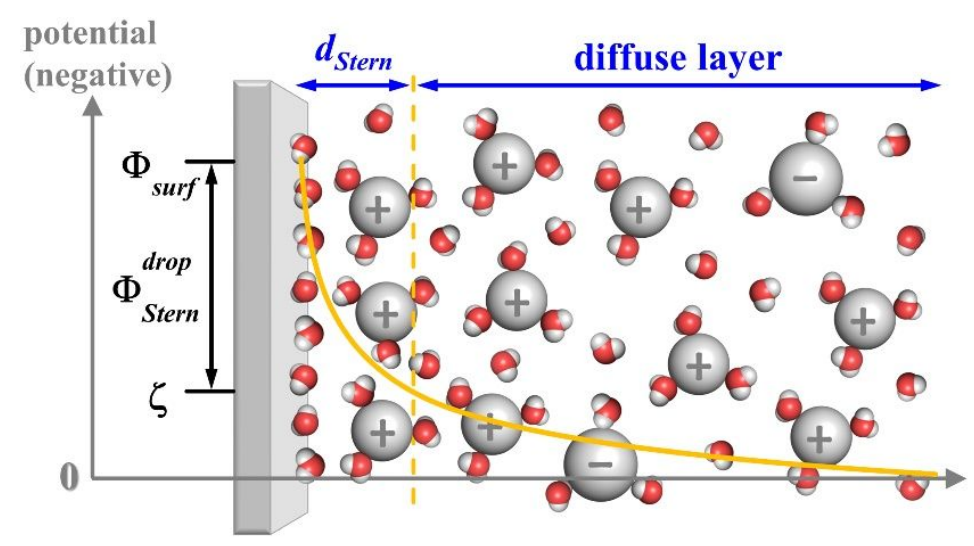

Figure S9. Schematic figure of the water structure at the interface between an aqueous electrolyte solution and a negatively charged surface. The surface potential is plotted as described by the Gouy-Chapman-Stern model. 
Table S7. The relationship between the depth monitored by SFG and the vibrational relaxation time of the ssp intensity decay with $\omega_{\text {pump }}=\omega_{\text {probe }}=\sim 3200 \mathrm{~cm}^{-1}$.

\begin{tabular}{ccccc}
\hline Surface & Condition & Thickness & $\mathrm{T}_{1}(\mathrm{fs})$ & Ref. \\
\hline Silica $/ \mathrm{H}_{2} \mathrm{O}$ & $\mathrm{pH}=5.7$ & diffuse layer & $\sim 300$ & 41 \\
Silica/ $/ \mathrm{H}_{2} \mathrm{O}$ & $\mathrm{pH}=12$ & diffuse layer & $255 \pm 25$ & 35 \\
Silica $/ \mathrm{H}_{2} \mathrm{O}$ & $\mathrm{pH}=2$ & $1^{\text {st }}$ monolayer & $570 \pm 30$ & 35 \\
$\mathrm{Silica} / \mathrm{H}_{2} \mathrm{O}$ & $\mathrm{pH}=6,0 \sim 1 \mathrm{mM} \mathrm{NaCl}$ & diffuse layer & $<200$ & 42 \\
$\mathrm{Silica} / \mathrm{H}_{2} \mathrm{O}$ & $\mathrm{pH}=6,0.5 \mathrm{M} \mathrm{NaCl}$ & $1^{\text {st }}$ monolayer, $<0.3 \mathrm{~nm}$ & $\sim 570$ & 42 \\
$\mathrm{Silica} / \mathrm{H}_{2} \mathrm{O}$ & $\mathrm{pH}=2,0 \mathrm{M} \mathrm{NaCl}$ & $1^{\text {st }}$ monolayer, $<0.3 \mathrm{~nm}$ & $577 \pm 140$ & 36 \\
Silica$/ \mathrm{H}_{2} \mathrm{O}$ & $\mathrm{pH}=2,0.1 \mathrm{M} \mathrm{NaCl}$ & $1^{\text {st }}$ monolayer, $<0.3 \mathrm{~nm}$ & $422 \pm 120$ & 36 \\
Silica$/ \mathrm{H}_{2} \mathrm{O}$ & $\mathrm{pH}=6,0.1 \mathrm{M} \mathrm{NaCl}$ & $1^{\text {st }}$ monolayer, $<0.3 \mathrm{~nm}$ & $633 \pm 88$ & 36 \\
Air $/ \mathrm{H}_{2} \mathrm{O}$ & $\mathrm{pH}=7$ & diffuse layer & 190 & 43 \\
Air $/ \mathrm{H}_{2} \mathrm{O}$ & $\mathrm{pH}=7$ & diffuse layer & 190 & 44 \\
$\mathrm{Lipid} / \mathrm{H}_{2} \mathrm{O}$ & $\mathrm{Lipid}: \mathrm{DMPS}$ & diffuse layer & $<100$ & 44 \\
$\mathrm{Lipid} / \mathrm{H}_{2} \mathrm{O}$ & DMPS, DPTAP, DPPC, DPPE & diffuse layer & $<80$ & 37 \\
$\mathrm{Bulk} \mathrm{H}_{2} \mathrm{O}$ & & & $\sim 200$ & 45,46 \\
\hline
\end{tabular}

\section{The vibrational dynamics of dangling $\mathrm{OH}$ at the PTFE/air surface.}

Figure S10 shows the ssp intensity decay of the dangling OH band at the PTFE/air surface ( $\sim 40 \%$ R.H.) with $\omega_{\text {pump }}=\omega_{\text {probe }}=\sim 3680 \mathrm{~cm}^{-1}$, and its vibrational relaxation time $\left(\mathrm{T}_{1}\right)$ is determined to be $1.5 \pm 0.2 \mathrm{ps}$.

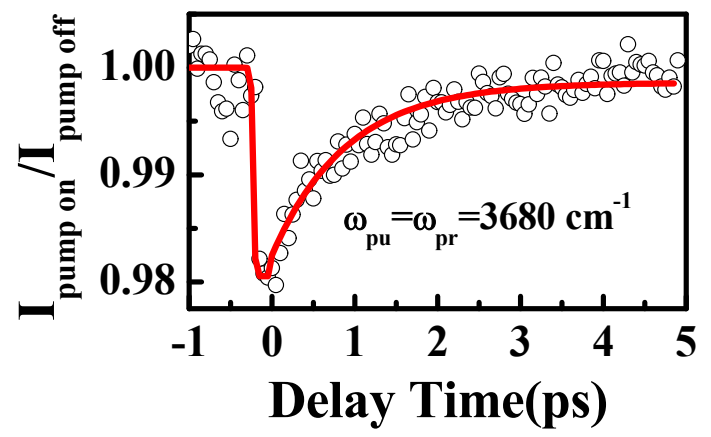

Figure S10. The ssp intensity decay of the dangling OH mode at the PTFE/air surface $(\sim 40 \%$ R.H.) after pumping (pu) and probing (pr) at frequencies of $\omega_{\mathrm{pu}}=\omega_{\mathrm{pr}}=3680 \mathrm{~cm}^{-1}$.

\section{References}

(1) Shen, Y. R. The Principles of Nonlinear Optics. Wiley, New York, 1984. 
(2) Lambert, A. G.; Davies, P. B.; Neivandt, D. J. Implementing the Theory of Sum Frequency Generation Vibrational Spectroscopy: A Tutorial Review. Appl. Spectrosc. Rev. 2005, 40, 103-145.

(3) Castellana, E. T.; Cremer, P. S. Solid Supported Lipid Bilayers: From Biophysical Studies to Sensor Design. Surf. Sci. Rep. 2006, 61, 429-444.

(4) Gopalakrishnan, S.; Liu, D.; Allen, H. C.; Kuo, M.; Shultz, M. J. Vibrational Spectroscopic Studies of Aqueous Interfaces: Salts, Acids, Bases, and Nanodrops. Chem. Rev. 2006, 106, $1155-1175$.

(5) Ye, S. J.; Nguyen, K. T.; Le Clair, S. V.; Chen, Z. In Situ Molecular Level Studies on Membrane Related Peptides and Proteins in Real Time Using Sum Frequency Generation Vibrational Spectroscopy. J. Struct. Biol. 2009, 168, 61-77.

(6) Tan, J. J.; Luo, Y.; Ye, S. J. A Highly Sensitive Femtosecond Time-Resolved Sum Frequency Generation Vibrational Spectroscopy System with Simultaneous Measurement of Multiple Polarization Combinations. Chin. J. Chem. Phys. 2017, 30, 671-677.

(7) Tan, J. J.; Zhang, B. X.; Luo, Y.; Ye, S. J. Ultrafast Vibrational Dynamics of MembraneBound Peptides at the Lipid Bilayer/Water Interface. Angew. Chem. Int. Ed. 2017, 56, 1297712981.

(8) Tan, J. J.; Zhang, J. H.; Li, C. Z.; Luo, Y.; Ye, S. J. Ultrafast Energy Relaxation Dynamics of Amide I Vibrations Coupled with Protein-Bound Water Molecules. Nat. Commun. 2019, 10, 1010 .

(9) Wei, X.; Shen, Y. R. Motional Effect in Surface Sum-Frequency Vibrational Spectroscopy. Phys. Rev. Lett. 2001, 86, 4799-4802.

(10) Tyrode, E.; Johnson, C. M.; Baldelli, S.; Leygraf, C.; Rutland, M. W. A Vibrational Sum Frequency Spectroscopy Study of the Liquid-Gas Interface of Acetic Acid-Water Mixtures: 2. Orientation Analysis. J. Phys. Chem. B 2005, 109, 329-341.

(11) Gan, W.; Wu, D.; Zhang, Z.; Feng, R.; Wang, H. Polarization and Experimental Configuration Analyses of Sum Frequency Generation Vibrational Spectra, Structure, and Orientational Motion of the Air/Water Interface. J. Chem. Phys. 2006, 124, 114705-114705.

(12) Zhuang, X.; Miranda, P. B.; Kim, D.; Shen, Y. R. Mapping Molecular Orientation and Conformation at Interfaces by Surface Nonlinear Optics. Phys. Rev. B 1999, 59, 12632-12640.

(13) Yang, M. K.; French, R. H.; Tokarsky, E. W. Optical Properties of Teflon ${ }^{\circledR}$ AF Amorphous Fluoropolymers. J. Micro/Nanolithogr., MEMS, MOEMS 2008, 7, 033010.

(14) Murphy, W. F. The Rovibrational Raman Spectrum of Water Vapour $\mathrm{v}_{1}$ and $\mathrm{v}_{3}$. Mol. Phys. 1978, 36, 727-732. 
(15) Sun, S.; Tang, F.; Imoto, S.; Moberg, D. R.; Ohto, T.; Paesani, F.; Bonn, M.; Backus, E. H. G.; Nagata, Y. Orientational Distribution of Free O-H Groups of Interfacial Water is Exponential. Phys. Rev. Lett. 2018, 121, 246101.

(16) Page, R. H.; Frey, J. G.; Shen, Y. R.; Lee, Y. T. Infrared Predissociation Spectra of Water Dimer in a Supersonic Molecular Beam. Chem. Phys. Lett. 1984, 106, 373-376.

(17) Li, Z. G.; Xu, J. W.; Jia, H. Q.; Hu, N. H. Observation of a Water-Anion Layer Constructed from Water Tetramers and Nitrate Anions in the Crystal Host of a Cobalt Complex. Inorg. Chem. Commun. 2006, 9, 969-972.

(18) Mukhopadhyay, U.; Bernal, I. Self-Assembled Hexameric Water Clusters Stabilized by a Cyano-Bridged Trimetallic Complex. Cryst. Growth Des. 2005, 5, 1687-1689.

(19) Huisken, F.; Kaloudis, M.; Kulcke, A.; Voelkel, D. IR Spectroscopy of Size-Selected Clusters at $3 \mu \mathrm{m}$ Wavelength. Infrared Phys. Technol. 1995, 36, 171-178.

(20) Huisken, F.; Kaloudis, M.; Kulcke, A. Infrared Spectroscopy of Small Size-Selected Water Clusters. J. Chem. Phys. 1996, 104, 17-25.

(21) Paul, J. B.; Collier, C. P.; Saykally, R. J.; Scherer, J. J.; O'Keefe, A. Direct Measurement of Water Cluster Concentrations by Infrared Cavity Ringdown Laser Absorption Spectroscopy. J. Phys. Chem. A 1997, 101, 5211-5214.

(22) Douberly, G. E.; Miller, R. E.; Xantheas, S. S. Formation of Exotic Networks of Water Clusters in Helium Droplets Facilitated by the Presence of Neon Atoms. J. Am. Chem. Soc. 2017, 139, 4152-4156.

(23) Moudens, A.; Georges, R.; Goubet, M.; Makarewicz, J.; Lokshtanov, S. E.; Vigasin, A. A. Direct Absorption Spectroscopy of Water Clusters Formed in a Continuous Slit Nozzle Expansion. J. Chem. Phys. 2009, 131, 204312.

(24) Ceponkus, J.; Uvdal, P.; Nelander, B. Water Tetramer, Pentamer, and Hexamer in Inert Matrices. J. Phys. Chem. A 2012, 116, 4842-4850.

(25) Shukla, M. Raman Spectroscopic Study of Water Tetramer in [Cu(3aminomethyl)pyridine $\mathrm{OH}_{2}$ oxalate $\left.\cdot 2 \mathrm{H}_{2} \mathrm{O}\right]_{\mathrm{n}}$. Polyhedron 2016, 105, 200-204.

(26) Burnham, C. J.; Xantheas, S. S.; Miller, M. A.; Applegate, B. E.; Miller, R. E. The Formation of Cyclic Water Complexes by Sequential Ring Insertion: Experiment and Theory. J. Chem. Phys. 2002, 117, 1109-1122.

(27) Fajardo, M. E.; Tam, S. Observation of the Cyclic Water Hexamer in Solid Parahydrogen. J. Chem. Phys. 2001, 115, 6807-6810.

(28) Nicolaisen, F. M. IR Absorption Spectrum (4200-3100 $\left.\mathrm{cm}^{-1}\right)$ of $\mathrm{H}_{2} \mathrm{O}$ and $\left(\mathrm{H}_{2} \mathrm{O}\right)_{2}$ in $\mathrm{CCl}_{4}$. Estimates of the Equilibrium Constant and Evidence that the Atmospheric Water Absorption 
Continuum is Due to the Water Dimer. J. Quant. Spectrosc. Radiat. Transfer 2009, 110, 20602076.

(29) Prell, J. S.; Williams, E. R. Structures of Thermal, Mass-Selected Water Clusters Probed with Hydrophobic Ion Tags and Infrared Photodissociation Spectroscopy. J. Am. Chem. Soc. 2009, 131, 4110-4119.

(30) Kristinaitytė, K.; Dagys, L.; Kausteklis, J.; Klimavicius, V.; Doroshenko, I.; Pogorelov, V.; Valevičienè, N. R.; Balevicius, V. NMR and FTIR Studies of Clustering of Water Molecules: From Low-Temperature Matrices to Nano-Structured Materials Used in Innovative Medicine. J. Mol. Liq. 2017, 235, 1-6.

(31) Hirabayashi, S.; Yamada, K. M. T. Infrared Spectra and Structure of Water Clusters Trapped in Argon and Krypton Matrices. J. Mol. Struct. 2006, 795, 78-83.

(32) Watanabe, Y.; Maeda, S.; Ohno, K. Intramolecular Vibrational Frequencies of Water Clusters $\left(\mathrm{H}_{2} \mathrm{O}\right)_{\mathrm{n}}(\mathrm{n}=2-5)$ : Anharmonic Analyses Using Potential Functions Based on the Scaled Hypersphere Search Method. J. Chem. Phys. 2008, 129, 074315.

(33) Lee, H. M.; Suh, S. B.; Lee, J. Y.; Tarakeshwar, P.; Kim, K. S. Structures, Energies, Vibrational Spectra, and Electronic Properties of Water Monomer to Decamer. J. Chem. Phys. 2000, 112, 9759-9772.

(34) Samala, N. R.; Agmon, N. Temperature Dependence of Intramolecular Vibrational Bands in Small Water Clusters. J. Phys. Chem. B 2019, 123, 9428-9442.

(35) Eftekhari-Bafrooei, A.; Borguet, E. Effect of Surface Charge on the Vibrational Dynamics of Interfacial Water. J. Am. Chem. Soc. 2009, 131, 12034-12035.

(36) Tuladhar, A.; Dewan, S.; Pezzotti, S.; Brigiano, F. S.; Creazzo, F.; Gaigeot, M. P.; Borguet, E. Ions Tune Interfacial Water Structure and Modulate Hydrophobic Interactions at Silica Surfaces. J. Am. Chem. Soc. 2020, 142, 6991-7000.

(37) Bonn, M.; Bakker, H. J.; Ghosh, A.; Yamamoto, S.; Sovago, M.; Campen, R. K. Structural Inhomogeneity of Interfacial Water at Lipid Monolayers Revealed by Surface-Specific Vibrational Pump-Probe Spectroscopy. J. Am. Chem. Soc. 2010, 132, 14971-14978.

(38) Sudera, P.; Cyran, J. D.; Deiseroth, M.; Backus, E. H. G.; Bonn, M. Interfacial Vibrational Dynamics of Ice $\mathrm{I}_{\mathrm{h}}$ and Liquid Water. J. Am. Chem. Soc. 2020, 142, 12005-12009.

(39) Nibbering, E. T. J.; Elsaesser, T. Ultrafast Vibrational Dynamics of Hydrogen Bonds in the Condensed Phase. Chem. Rev. 2004, 104, 1887-1914.

(40) Lawrence, C. P.; Skinner, J. L. Vibrational Spectroscopy of HOD in Liquid $\mathrm{D}_{2} \mathrm{O}$. VII. Temperature and Frequency Dependence of the OH Stretch Lifetime. J. Chem. Phys. 2003, 119, 3840-3848. 
(41) Mcguire, J. A.; Shen, Y. R. Ultrafast Vibrational Dynamics at Water Interfaces. Science 2006, 313, 1945-1948.

(42) Eftekharibafrooei, A.; Borguet, E. Effect of Electric Fields on the Ultrafast Vibrational Relaxation of Water at a Charged Solid-Liquid Interface as Probed by Vibrational Sum Frequency Generation. J. Phys. Chem. Lett. 2011, 2, 1353-1358.

(43) Smits, M.; Ghosh, A.; Sterrer, M.; Müller, M.; Bonn, M. Ultrafast Vibrational Energy Transfer between Surface and Bulk Water at the Air-Water Interface. Phys. Rev. Lett. 2007, 98, 098302.

(44) Ghosh, A.; Smits, M.; Bredenbeck, J.; Bonn, M. Membrane-Bound Water is Energetically Decoupled from Nearby Bulk Water: An Ultrafast Surface-Specific Investigation. J. Am. Chem. Soc. 2007, 129, 9608-9609.

(45) Lock, A. J.; Woutersen, S.; Bakker, H. J. Ultrafast Energy Equilibration in HydrogenBonded Liquids. J. Phys. Chem. A 2001, 105, 1238-1243.

(46) Cowan, M. L.; Bruner, B. D.; Huse, N.; Dwyer, J. R.; Chugh, B.; Nibbering, E. T. J.; Elsaesser, T.; Miller, R. J. D. Ultrafast Memory Loss and Energy Redistribution in the Hydrogen Bond Network of Liquid $\mathrm{H}_{2} \mathrm{O}$. Nature 2005, 434, 199-202. 